\title{
La organización de la enseñanza de la lectura en las aulas chilenas. Diferencias entre profesores calificados como básicos o como competentes*
}

\author{
The Organization of Reading Instruction in Classrooms in Chile. \\ Some Differences between Basic and Qualified Teachers as Competent
}

Recibido: 31 de julio de 2012 | Revisado: 10 de agosto de 2014 | Aceptado: 21 de octubre de 2014

\author{
CAROlina ItUrRa Herrera ** \\ Universidad de Talca, Chile
}

doi:10.11144/Javeriana.upsy14-2.oela

Para citar este artículo: Iturra, C. (2015). La organización de la enseñanza de la lectura en las aulas chilenas. Diferencias entre profesores calificados como básicos o como competentes. Universitas Psychologica, 14(2), 535-548. http://dx.doi.org.10.11144/ Javeriana.upsy14-2.oela

* El estudio fue realizado gracias a los fondos del proyecto Fondecyt (Fondo Nacional de Desarrollo Científico y Tecnológico) $\mathrm{N}^{\circ} 11110158$. Se agradece también, el apoyo del Centro de Estudios del Ministerio de Educación y de CPEIP, quienes dieron acceso al material incluido en el estudio.

** Doctora en Psicología Educacional y Profesora de la Facultad de Psicología de la Universidad de Talca, Chile. Su trabajo de investigación se centra en el análisis de la práctica educativa, las metodologías innovadoras y la formación inicial docente. Correo electrónico: citurra@utalca.cl

\section{RESUMEN}

El artículo describe las prácticas educativas de profesores chilenos en clases de comprensión de textos en educación primaria, seleccionadas del sistema de evaluación docente. Utilizando un sistema de análisis del discurso en el aula, se segmentó cada una de las tareas que fueron desplegadas en ellas. Esto permitió describir la organización de la enseñanza, identificando patrones asociados a cada fase de la lectura. Se observaron algunas diferencias de acuerdo a la calificación otorgada a los profesores, siendo los profesores calificados como "competentes" quienes organizaron de manera más coherente las actividades, incluyendo mayores acciones de cierre. No obstante, se desempeñaron de manera similar al resto de los profesores en la fase during-reading, no logrando desplegar acciones tendientes a supervisar la comprensión.

Palabras clave

análisis de la práctica educativa; comprensión de textos; evaluación desempeño docente

\footnotetext{
A B S T R A C T

The article describes Chilean teachers' educational practice in primary education reading comprehension classes, selected from the Chilean teacher evaluation system. Each task deployed were segmented using a classroom discourse analysis system. This allowed to describe the organization of teaching, identifying patterns associated with each reading phase. Some differences according to the rating of teachers were observed; teachers qualified as "competent" organized activities more consistently, including more closing actions. However, they performed similarly to the rest of the teachers in the during-reading phase, failing in deploying actions to monitoring comprehension.

Keywords

Educational practice analysis; text comprehension; teacher performance evaluation
} 


\section{Introducción}

La competencia lectora es un proceso progresivo que se incrementa en la medida en que los lectores van apropiándose de mayores conocimientos y estrategias, facilitando así el entendimiento de los textos con los cuales interactúan (Chall, 1996). Así, los lectores expertos son capaces de desplegar estrategias cognitivas y metacognitivas que favorecen el entendimiento de las ideas contenidas en los textos, permitiéndoles construir significados en los que dialoga la intención del autor con los propios conocimientos del lector (Kintsch, 1998; Kintsch $\&$ Kintsch, 2005).

Este proceso complejo requiere de estrategias cognitivas que los lectores ponen en marcha como la activación de conocimientos previos, el establecimiento de coherencias locales y globales, la utilización de inferencias, entre otros, para así lograr construir representaciones mentales de los textos leídos (Dole, Nokes, \& Drits, 2009), así como estrategias metacognitivas tendientes a dirigir mentalmente el proceso mediante el establecimiento de propósitos para la lectura, la supervisión de la misma y la evaluación del nivel de comprensión alcanzado (Block \& Pressley, 2002; Pressley \& Afflerbach, 1995; Sánchez, García, \& Rosales, 2010). Al respecto, la investigación ha puesto en evidencia la necesidad de que los lectores sean capaces de monitorear constantemente el conocimiento que van construyendo. Así por ejemplo, Tobias y Everson (2000) identificaron relaciones significativas entre el monitoreo de la evaluación del conocimiento y el rendimiento en pruebas de comprensión de textos y matemáticas, indicando que cuanto más estratégicos son los estudiantes al monitorear su comprensión, los logros académicos alcanzados son mayores. En este mismo sentido, se reconoce la importancia del papel que juegan los procesos inferenciales al permitir conexiones entre el conocimiento previo y la información del texto, además de completar información faltante del texto, necesaria para acceder a la representación del mismo (Anderson \& Pearson, 1984; Belinchón, Rivière, \& Igoa, 2000). Además, se sostiene que estas inferencias se podrían activar en el transcurso de la lectura o comprensión (on-line), mientras que otras lo harían posteriormente, durante la tarea de recuperación (off-line) (León \& Pérez, 2000 citado en León, 2001). Ambos procesos, así como las estrategias desplegadas por los lectores, les permitirían lograr comprender lo que leen.

Sin embargo, para alcanzar este objetivo en las aulas se requiere de un trabajo de colaboración entre profesor y estudiantes, orientado a generar estrategias que le permitan a estos últimos lograr una adecuada competencia lectora. Desde esta mirada, situamos los procesos de aprendizaje de la comprensión de textos en el contexto del aula, asumiendo que esta representa un espacio social y cultural (Vygostky, 1978), en la que se logra crear una intersubjetividad, permitiendo a los participantes de la interacción social compartir significados, informaciones, referencias y conocimientos que facilitarían y propiciarían la comprensión (Edwards \& Mercer, 1987). Al respecto, Wells (2001) plantea que las actividades en las aulas son acciones conjuntas que llevan a cabo profesores y alumnos, organizándose de acuerdo a una jerarquía que abarcaría desde acciones globales (unidades didácticas) hasta acciones más específicas, como las rutinas escolares. Además, cada una de estas acciones se organizaría en función del tipo de contenido instruccional (Stodolsky, 1991).

Considerando la naturaleza del contenido escolar de la comprensión de textos, en las últimas décadas se ha planteado una serie de modelos instruccionales orientados a lograr la comprensión de textos por parte de los estudiantes. Dentro de estos, destacamos the reciprocal teaching de Palincsar y Brown (1984), quienes proponen un marco instruccional sustentado en cuatro orientaciones estratégicas para la comprensión usadas antes, durante y después de la lectura de un texto. Dichas autoras sostienen que mediante el modelado y el pensamiento en voz alta, los profesores debieran enseñar a resumir, preguntar, clarificar y predecir. Siguiendo esta línea, Solé (2000) plantea una serie de estrategias didácticas, secuenciadas en distintos momentos a lo largo de la lectura de un texto. La primera de ellas, la fase de prelectura o anticipación, se orientaría a definir los objetivos 
de lectura e indagar en los conocimientos previos, para focalizar la atención hacia el texto y permitir acciones anticipatorias acerca de lo que se tratará el mismo. La segunda fase nominada durante la lectura o fase de construcción, focalizaría la atención en el contenido principal, mediante acciones tendientes a controlar la comprensión. Finalmente, la tercera fase después de la lectura o evaluación, se dirigiría a evaluar el proceso de comprensión a través del uso de diversos instrumentos, como resúmenes, mapas conceptuales, mapas mentales, entre otros. Considerando estas estrategias, los resultados de investigaciones realizadas por Duque y Correa (2012) y Duque y Ovalle (2011), estudiando interacciones en las aulas de educación inicial a través de una metodología mixta sustentada en el análisis del discurso y análisis de redes sociales, han identificado que las profesoras que se orientaron a trabajar tareas cognitivas de mayor demanda focalizando el trabajo en la elaboración de los aspectos implícitos del texto, lograron una mayor elaboración inferencial en sus estudiantes, mientras que las que centraron la atención en los aspectos más explícitos de los textos alcanzaron un menor grado de elaboración y porcentajes menores de inferencias en sus alumnos. Por tanto, se requerirían interacciones profesor-alumnos orientadas a construir espacios de negociación de significados que demanden una alta complejidad cognitiva para los estudiantes, para que estos puedan formular y desplegar distintos tipos de procesos inferenciales, facilitando así la comprensión.

Otro de los modelos que plantea la necesidad de fomentar procedimientos instruccionales para la enseñanza de estrategias de comprensión lectora es el elaborado por Clark y Graves (2005), quienes han identificado distintos tipos de andamios o scaffolding denominados marcos de instrucción, que se relacionarían con el diseño de la experiencia de lectura. De esta forma, sostienen que la planificación de actividades debiera incluir tareas que recogieran múltiples estrategias de comprensión, secuenciándolas de acuerdo a las fases de la lectura. Así, en la fase de prereading, se debieran incluir actividades tales como: activación y construcción de un contexto de conocimiento, entregar informa- ción sobre el tipo de texto que se va a leer, palabras y conceptos claves, cuestionamientos orientados a predecir la lectura, entre otras. En la fase during Reading, las actividades propuestas por los autores serían lectura silenciosa, lectura oral de los estudiantes, modificaciones al texto, etc. Finalmente, la fase postreading implicaría el desarrollo de tareas de cuestionamiento, discusión y construcción de conectores. A partir de lo anterior, Liang, Peterson y Graves (2005), investigaron los efectos de la instrucción en Scaffolded Reading Experience (SER). Mediante un estudio cuasiexperimental. identificaron que los estudiantes que recibieron instrucción bajo el marco de la SRE, lograron un rendimiento significativamente más alto en pruebas de selección múltiple de comprensión de textos que los estudiantes que recibieron instrucción bajo otro enfoque. Esto implicaría, para los autores, la necesidad de estructurar las experiencias de lectura a partir de procedimientos didácticos que actúen como andamios del proceso comprensivo.

En esta misma línea, los hallazgos efectuados por Rosales, Iturra, Sánchez y De Sixte (2006), Sánchez et al. (2010), Sánchez, Rosales, García, De Sixte y Castellano (2008), quienes han indagado cómo se articulan actividades típicas de lectura conjunta, mediante un sistema de análisis sustentado desde una teoría del cómo comprendemos, han logrado identificar ciertos patrones de actividades típicas (ATA), entendiendo por actividad típica al conjunto de acciones regulares que suelen repetirse a lo largo de una unidad, que poseen un propósito distintivo y un plan de trabajo reconocido. Estas ATA, dependiendo de la secuenciación temporal de sus tareas y del tipo de diálogo propiciado, encarnarían ciertos contextos de trabajo, propicios o no, para el desarrollo del proceso de aprendizaje de la comprensión de textos. En este sentido, algunos trabajos (Ortiz et al., 2007; Rincón, De la Rosa, Chois, $\&$ Niño, 2003) han identificado ciertos formatos instruccionales, observando cierta disposición de parte de los profesores, para orientar el trabajo a la comprensión de textos. Sin embargo, las actividades y tareas planificadas y desplegadas en las aulas no son suficientes o carecen de propósitos claros para lograr aprendizajes significativos. En este sentido, 
las conclusiones de tales investigaciones advierten la importancia de incluir tareas que permitan preparar el proceso, hacer seguimiento del mismo y finalmente evaluar los aprendizajes alcanzados.

A nivel nacional, el Ministerio de Educación Chileno (Mineduc) ha desarrollado en los últimos años, una serie de acciones tendientes a mejorar la inclusión de estos principios y estrategias metodológicas. Por un lado, las orientaciones didácticas (Mineduc, 2009a), descritas en los programas de estudio del sector curricular de lenguaje y comunicación, que en el caso de la lectura proponen una serie de actividades que debieran ser trabajadas e incluidas en las experiencias de aprendizaje. Por otro, la política de textos escolares (Mineduc, 2001 citado en Mineduc, 2009b) ha incorporado, como parte de las actividades de los libros de textos, orientaciones para incluir tareas vinculadas a los momentos antes, durante y después de la lectura. No obstante, existen pocos trabajos que analicen y describan las prácticas docentes que abordan la enseñanza de la comprensión de textos en las aulas. Así por ejemplo, Rosas y Jiménez (2009) mediante una aproximación cualitativa, utilizando registros etnográficos, analizaron clases en las que se trabajaba la comprensión de textos, en los grados escolares de sexto y octavo de primaria. A partir del análisis de las observaciones, constataron que existía poca variabilidad en las actividades incluidas en las aulas, estableciendo que parte importante de la planificación no consideraba el trabajo específico en torno a las estrategias de aprendizaje. Además, la mayoría de las actividades eran extraídas directamente de los textos escolares, sin necesariamente revisar las respuestas elaboradas por los estudiantes.
Es partir de la necesidad de describir la forma en que se organiza y estructura el proceso de aprendizaje en torno a la comprensión de textos, que la presente investigación busca caracterizar la interacción profesor-alumno, en 30 sesiones grabadas y pertenecientes al material audiovisual del Sistema de Evaluación Profesional Docente, para poder describirlas y establecer algunas diferencias a partir de la calificación otorgada y asociada al desempeño de los profesores.

\section{Método}

\section{Participantes}

Se seleccionaron 30 grabaciones de un total de 538 sesiones pertenecientes al sistema de evaluación del desempeño docente chileno del año 2008. Estas grabaciones consisten en sesiones de 40 minutos de clases, en las que participan los profesores evaluados y sus estudiantes, por tanto, se seleccionaron 30 clases. Las grabaciones forman parte del proceso de evaluación del desempeño docente, llevado a cabo por el Centro de Perfeccionamiento, Experimentación e Investigaciones Pedagógicas (CPEIP), organismo gubernamental dependiente del Ministerio de Educación Chileno, que es el encargado de gestionar dicha evaluación y que es obligatoria para todos los profesores del sector municipal o público. Estas grabaciones corresponden al módulo 2 del instrumento de evaluación denominado portafolio (Mineduc, 2004). Para seleccionarlas, se establecieron criterios muestrales intencionales (Ruiz, 2012), orientados principalmente a que la sesiones

TABLA 1

Caracterización de la muestra

\begin{tabular}{lccccc}
\hline & \multicolumn{5}{c}{ Nivel de enseñanza (grados) } \\
\hline Evaluación desempeño docente & 5 grado & 6 grado & 7 grado & 8 grado & total \\
\hline Básico & 6 & 3 & 6 & 1 & 15 \\
Competente & 5 & 3 & 5 & & 13 \\
Destacado & 1 & & 1 & & 2 \\
Total & 11 & 6 & 12 & 1 & 30 \\
\hline
\end{tabular}

Fuente: elaboración propia 
incluyeran la comprensión de textos como contenido escolar y que provinieran del nivel escolar de segundo ciclo de primaria. Este ciclo educativo se organiza en función de 4 grados escolares, $5 .{ }^{\circ}, 6^{\circ}$, 7. $\mathrm{y} 8 .^{\circ}$ y las edades de los estudiantes oscilan entre los 10-14 años. Se accedió además a los niveles de desempeño docente obtenidos por los profesores, tal y como se muestra en la Tabla 1.

\section{Sistema de análisis}

El sistema que guió el análisis de las sesiones es el propuesto por Sánchez et al. (2010), quienes plantean distintas unidades de análisis que abordarían el fenómeno desde distintas miradas, logrando representar y describir, en diferentes niveles, cómo profesores y estudiantes trabajan conjuntamente y cuáles son finalmente las actividades, tareas y procesos que se desarrollan y activan, en el transcurso de un proceso de enseñanza-aprendizaje dedicado a la comprensión de textos. Dichas unidades tienen como propósito segmentar la interacción discursiva entre los participantes. Así, el sistema se compone a partir de 5 unidades de análisis, dos de ellas, las (1) Unidades Didácticas y (2) Sesiones, permiten la segmentación considerando criterios temporales (número de sesiones programadas por unidad, tiempo de duración de cada sesión) establecidos por el profesor o por los centros. En tanto que las (3) Actividades Típicas (4) Episodios y (5) Ciclos seleccionarían y analizarían las interacciones verbales, a partir de criterios teóricos y metodológicos.

Particularmente, en esta investigación, se utilizaron como unidades de análisis, las actividades típicas de lectura conjunta y los episodios. Las primeras definidas como conjuntos de acciones regulares que suelen repetirse a lo largo de una unidad, que poseen un propósito distintivo y un plan de trabajo reconocido, mientras que los segundos, se conceptualizan como conjuntos de intercambios verbales entre profesores y estudiantes que poseen un objetivo o meta común y que suelen adoptar modos de participación característicos. Este sistema cuenta con un procedimiento declarado (Sánchez et al., 2008) que opera desfragmentando la interacción comunicativa en el aula. Respecto a la fiabilidad del sistema de análisis, las puntuaciones obtenidas en el procedimiento de juicio experto han arrojado, para las unidades de análisis, un coeficiente de 0.9 episodios y 0.86 para los ciclos, mientras que en las dimensiones de análisis, se han obtenido puntuaciones de 0.86 para las categorías de análisis y 0.78 para los contenidos públicos.

\section{Procedimiento}

En primer lugar, se seleccionó la muestra utilizando los criterios establecidos para la misma. Una vez seleccionada, se procedió a la transcripción literal de las sesiones. En segundo lugar, se segmentó el discurso identificando las actividades típicas de lectura, empleando como criterios de división: las acciones enmarcadas por un propósito central y los patrones de actuación característicos. Cabe señalar que aquellos segmentos de interacción que no se orientaban al trabajo en comprensión de textos, no fueron analizados. En tercer lugar, se segmentaron las actividades típicas, identificándose los episodios que las constituyeron. Para ello, se utilizaron como criterios de división dos aspectos: el primero vinculado a la identificación del objetivo específico de cada episodio y el segundo relacionado al reparto de roles característicos en cada uno.

\section{Análisis de datos}

Para establecer la organización y secuenciación de la enseñanza considerando las tres fases de la lectura (Clark \& Graves, 2005; Solé, 2000), se relacionaron las metas de cada fase con las metas de cada episodio, para posteriormente agrupar los episodios con cada fase. En este sentido, todos los episodios ubicados temporalmente antes de la ejecución de la lectura, corresponderían al momento antes de la lectura. Para el momento durante la lectura, además de la acción procedimental de la misma, se incluyeron todos los episodios que se articulaban con esta acción, lo que implicaba la articulación entre los episodios con el propósito de supervisar las ideas o vocabulario del texto (por ejemplo, lectura de un trozo del texto y revisión del significado de una palabra que forma parte 
del texto). Para el momento después de la lectura, se agruparon aquellos episodios ubicados temporalmente al término de la fase durante la lectura. Posteriormente, se contabilizaron las frecuencias de cada episodio y se calcularon los porcentajes considerando el total de episodios. En segundo lugar, para la identificación de los patrones por cada fase, se analizaron y agruparon los tipos de episodio desplegados en cada fase y la temporalidad de las acciones. En tercer lugar, para establecer las diferencias considerando el desempeño obtenido por los profesores, se conformaron dos grupos. El primero reunió a la totalidad de profesores calificados como básicos y el segundo a los profesores calificados como competentes, sumándose a estos los dos profesores calificados como destacados. Se consideraron como medidas de contrastación, el tipo de patrón identificado.

\section{Aspectos éticos}

Las grabaciones se solicitaron formalmente al CPEIP, firmando un acuerdo de confidencialidad que se encuentra normado. Adicionalmente, como parte del protocolo para resguardar información sensible, una vez transcritas cada una de las sesiones, se le asignó un número a cada una, para asociar los antecedentes de la clase a ese número asignado. El resto de la información como la calificación obtenida en la evaluación, fue vinculada con el número asignado a cada clase y transcripción. Por otro lado, de acuerdo a los principios éticos y código de conducta para los psicólogos propuesto por la American Psychological Association (APA), se describe en el estándar 8 de investigación y publicación, que los psicólogos pueden prescindir del consentimiento informado cuando estudian prácticas educativas habituales, currículo o métodos de supervisión en las aulas aplicados a contextos educativos (APA, 2010).

\section{Resultados}

\section{Tipos de episodios}

A partir de los análisis efectuados, se identificó un total de 22 tipos de episodios. Cada uno de estos tipos se caracterizó por contar con una meta distintiva (Apéndice), además de propiciar ciertos patrones de actuaciones particulares que implicaron la asunción de roles característicos en el aula. No obstante, en algunos de ellos se detectaron diferencias relacionadas al patrón de actuación. Asî por ejemplo, los tipos de episodio de planificación fueron desplegados en todas las actividades típicas analizadas. La meta de estos consistía en explicitar el objetivo de la lectura, acción llevada en gran parte de los casos por la profesora, en tanto se esperaba que los estudiantes escucharan en silencio, prestando atención a las indicaciones de la profesora para lograr captar el objetivo de la lectura. Sin embargo, fue posible identificar que en algunas actividades típicas, los estudiantes participaron, mediante intervenciones dirigidas por los profesores, en la clarificación del propósito de la lectura.

Por otro lado, se observó que en ocasiones, algunos tipos de episodios se agruparon bajo un objetivo central, pero difirieron en los patrones de actuaciones, como en el caso de las configuraciones de realización de actividades que agrupaban tres tipos de episodios base: "instrucciones guía de trabajo en aula", "desarrollo guía de trabajo en aula" y "revisión guía de trabajo en aula". Estas configuraciones fueron identificadas habitualmente al final de las actividades típicas, y utilizadas por los profesores como una estrategia de trabajo después de la lectura del texto.

En relación con la frecuencia de cada tipo de episodio, considerando la calificación otorgada a los profesores en la evaluación de su desempeño, la Tabla 1 presenta los resultados de acuerdo a las frecuencias y porcentajes.

Los tipos de episodios más frecuentes en ambos grupos de profesores fueron los dedicados a planificar la actividad, activar conocimientos previos, leer, interpretar la lectura y recapitular la experiencia, entre otros. En tanto, los menos frecuentes resultaron aquellos dirigidos a la autoevaluación, instrucciones tarea, repaso de contenidos, etc. Aun cuando estas diferencias entre las frecuencias solo pudieran indicar qué tipos de episodios fueron más prototípicos, también permiten establecer ciertas distinciones. Así por ejemplo, en el caso de los tipos de episodios dirigidos 
TABLA 1

Resumen de frecuencias y porcentajes de representatividad de los tipos de episodios

\begin{tabular}{|c|c|c|c|c|}
\hline \multirow[t]{2}{*}{ Tipos de episodios } & \multicolumn{2}{|c|}{ Profesores calificados básicos } & \multicolumn{2}{|c|}{ Profesores calificados competentes } \\
\hline & $\begin{array}{r}\text { Frecuencia tipo } \\
\text { episodios x ATA }\end{array}$ & $\begin{array}{c}\% \\
\text { Representatividad }\end{array}$ & $\begin{array}{r}\text { Frecuencia tipo } \\
\text { episodios x ATA }\end{array}$ & $\begin{array}{c}\% \\
\text { Representatividad }\end{array}$ \\
\hline Lectura & 15 & 100 & 15 & 100 \\
\hline Planificación & 15 & 100 & 15 & 100 \\
\hline Conocimientos previos & 14 & 93 & 15 & 100 \\
\hline Interpretación & 10 & 67 & 15 & 100 \\
\hline Desarrollo guía de trabajo aula & 9 & 60 & 11 & 73 \\
\hline Instrucciones guía de trabajo aula & 9 & 60 & 12 & 80 \\
\hline Organización lectura & 9 & 60 & 12 & 80 \\
\hline Recapitulación & 9 & 60 & 10 & 67 \\
\hline Revisión guía de trabajo aula & 8 & 53 & 9 & 60 \\
\hline Vocabulario & 4 & 26 & 1 & 6.7 \\
\hline Análisis de experiencias & 3 & 20 & 2 & 13 \\
\hline Dictado & 1 & 6.7 & 1 & 6.7 \\
\hline $\begin{array}{l}\text { Elaboración conjunta guía de tra- } \\
\text { bajo }\end{array}$ & 1 & 6.7 & 1 & 6.7 \\
\hline Instrucciones subrayado & 1 & 6.7 & ....... & 0 \\
\hline Mapa conceptual & 1 & 6.7 & 1 & 6.7 \\
\hline Autoevaluación & ...... & ....... & 2 & 13 \\
\hline Explicación contenido & ...... & ...... & 1 & 6.7 \\
\hline $\begin{array}{l}\text { Exposición oral y revisión de guía } \\
\text { de trabajo en aula }\end{array}$ & ....... & ....... & 2 & 13 \\
\hline Identificación estructura noticia & ....... & ....... & 1 & 6.7 \\
\hline Instrucciones tarea & ....... & ....... & 2 & 13 \\
\hline Repaso contenido & ....... & ...... & 1 & 6.7 \\
\hline Revisión tarea & ....... & ....... & 1 & 6.7 \\
\hline
\end{tabular}

Fuente: elaboración propia

a interpretar las ideas del texto, esto es, supervisar la comprensión de las ideas o estructuras de los mismos, se encontró una mayor presencia de estos en las acciones conducidas por los profesores calificados como competentes. De igual modo, la tarea de organizar la lectura estableciendo los pasos para enfrentar la misma, fue identificada en una mayor proporción en los profesores calificados como competentes.

De especial interés es el resultado del tipo de episodio de mapa conceptual, que permitiría organizar las ideas, estableciendo jerarquías en las mismas experiencias. Sin embargo, este tipo de episodio resultó ser uno de los de más baja frecuencia en ambos grupos de profesores.

\section{Los momentos de la lectura}

\section{Antes de la lectura}

Una de las características centrales detectadas en las 30 actividades típicas analizadas, es que todas correspondieron a un patrón guiado por planes (Sánchez et al., 2010), es decir, todas incluyeron un episodio de planificación que guiaba las tareas que se desarrollarían posteriormente. No obstante, fue posible identificar ciertas diferencias relacionadas con la organización, secuenciación e inclusión de otros episodios como conocimientos previos o revisión de tarea. 
En general, se detectó una mayor presencia de una forma de organización que iniciaba la actividad con la planificación de la misma, seguido de una tarea de activación de conocimientos previos. Sin embargo, en algunas ocasiones se detectó un secuenciación inversa, comenzando la actividad con un episodio de conocimientos previos, para proseguir con la planificación de la misma. En otras oportunidades, se detectó la inclusión de más de un episodio de conocimientos previos y/o de planificación intercalándose, u otros tipos de episodios.

Respecto a las diferencias de estas organizaciones entre profesores calificados como básicos y competentes (Tabla 2), se puede indicar que la tendencia de los profesores calificados como competentes fue iniciar la actividad típica de lectura conjunta con una organización única de episodio de planificación seguido por otro de conocimientos previos.

En tanto que los profesores calificados como básicos desplegaron una mayor variabilidad en las formas de organización de esta fase, identificándose dos maneras más frecuentes. La primera que seguía el mismo patrón de los profesores calificados como competentes (organización 1) y la segunda en que intercalaron en más de una oportunidad, episodios de planificación y/ o conocimientos previos. Es decir, en esta última organización, se repetían en varias oportunidades, acciones destinadas a explicitar la meta o propósito de la actividad y acciones orientadas a evocar conocimientos previos.

\section{Durante la lectura}

Se detectaron tres modos de organizar y secuenciar los episodios de esta fase (Tabla 3). Por un lado, episodios únicos de lectura en los que se agregaba, en algunas oportunidades, un episodio dirigido a organizar la misma. Por el otro, episodios de lectura seguidos de otros tipos de episodios como vocabulario, interpretación, entre otros, secuenciados de manera lineal. Finalmente, episodios de lectura intercalados con episodios de interpretación, vocabulario o desarrollo de tareas. La diferencia entre

TABLA 2

Diferencias entre las organizaciones del momento antes de la lectura

\begin{tabular}{lcc}
\hline Organizaciones & $\begin{array}{c}\text { Profesores calificados } \\
\text { básicos }(\%)\end{array}$ & $\begin{array}{c}\text { Profesores calificados } \\
\text { competentes (\%) }\end{array}$ \\
\hline Org. 1 Planificación- conocimientos previos & 40 & 73 \\
Org. 2 Invertido conocimientos previos - planificación & 13 & 7 \\
Org. 3 Episodio único & 7 & $\ldots .$. \\
Org. 4 Intercalado de planificación-conocimientos previos & 33 & 7 \\
Org. 5 Inclusión otro tipo de episodios & 7 & 13 \\
\hline
\end{tabular}

Fuente: elaboración propia

TABLA 3

Diferencias entre las organizaciones del momento durante la lectura

\begin{tabular}{lcc}
\hline Organizaciones & $\begin{array}{c}\text { Profesores calificados } \\
\text { básicos (\%) }\end{array}$ & $\begin{array}{c}\text { Profesores calificados } \\
\text { competentes (\%) }\end{array}$ \\
\hline Lectura & 40 & 46 \\
Lectura más interpretación u otro episodio & 8 & 8 \\
Configuraciones & 52 & 46 \\
Lectura - interpretación & 46 & 38 \\
Lectura-vocabulario & 8 & 4 \\
Lectura- desarrollo guía trabajo & & 4 \\
\hline
\end{tabular}

Fuente: elaboración propia 
esta organización y la anterior, fue el número de veces en que se repitió esta acción de leer un trozo del texto y posteriormente interpretar o clarificar el significado de alguna palabra, configurándose de esta manera, una estrategia particular de supervisión de lo leído, que en algunas de las actividades típicas analizadas llegó a una frecuencia de repetición de 11 oportunidades.

Respecto a estas configuraciones, la mayor frecuencia en ambos grupos, fue aquella en que se articulaba un episodio de lectura seguido de un episodio de interpretación de la lectura. La segunda de menor frecuencia, se estructuraba a partir de un episodio de lectura y posteriormente un episodio de vocabulario. Finalmente, la tercera organización correspondía a una particular estrategia desplegada por una profesora calificada como competente, en la que se leía parte del texto y se respondía conjunta y públicamente, las preguntas contenidas en una guía de trabajo.

\section{Después de la lectura}

Una de las características más distintivas del desarrollo de esta fase, es que cerca del $70 \%$ de la muestra enfrentó el momento de profundización de la lectura a través de la resolución de guías de trabajo. Las otras actividades típicas organizaron la tarea de profundización, mediante el desarrollo de una tarea específica como la interpretación o un conjunto de tareas que no suponían la resolución de guías de trabajo.

Retomado la forma más característica de desarrollar esta fase, se puede indicar que, en general, estos patrones de actuaciones se configuraron a partir de la unión de tres episodios entremezclados entre sí. El primero de ellos dedicado a dar las instrucciones de la guía, acción realizada por los profesores de manera monologal o mediante diálogos con los estudiantes en los que habitualmente se revisaban conjuntamente las preguntas y/o se entregaban indicaciones para responderlas. El segundo, dedicado a desarrollar la guía, acción ejecutada por los estudiantes de forma individual o en pequeños grupos de trabajo, pudiendo ser supervisados o no por los profesores. Y el tercero, revisión de guía, se orientaba a evaluar las respuestas desarrolladas por los estudiantes, generalmente a través de un diálogo o de exposiciones orales de las respuestas por parte de los estudiantes. En algunas oportunidades, estas configuraciones incluían otro tipo de episodios dirigidos a interpretar las ideas del texto, mientras que en otras solo se observaba una parte de ellas, como un episodio de instrucciones sumado a uno de desarrollo.

Desde el punto de vista temporal, habitualmente eran iniciadas en el momento después de la lectura. Respecto a las diferencias observadas entre profesores calificados básicos y competentes, resumidos en la Tabla 4, se pudo identificar que indistintamente si la configuración se iniciaba durante o después de la lectura, o implicaba la inclusión de otro tipo de episodios, solo un $40 \%$ de los profesores calificados como básicos cerraron la configuración, es decir, desplegaron acciones explícitas orientadas a evaluar el trabajo desarrollado en las guías, en comparación con el 100 \% obtenido por los profesores calificados como competentes, que sí exhibieron, en todas las ocasiones, acciones tendientes a evaluar el trabajo.

Tabla 4

Diferencias entre las organizaciones del momento después de la lectura

\begin{tabular}{lcc}
\hline Organizaciones & $\begin{array}{c}\text { Profesores calificados } \\
\text { básico (\%) }\end{array}$ & $\begin{array}{c}\text { Profesores calificados } \\
\text { competentes (\%) }\end{array}$ \\
\hline Episodio único & 7 & 13 \\
Conjunto de episodio & 26 & 7 \\
Configuraciones realización guías trabajo aula. & 67 & 80 \\
Configuraciones completas & 40 & 100 \\
Configuraciones incompletas & 60 & \\
\hline
\end{tabular}

Fuente: elaboración propia 


\section{Discusión}

A modo de conclusión, se reconoce que los lectores expertos despliegan estrategias y procesos que les permiten ir incrementando su competencia lectora, favoreciendo así la comprensión de los textos que leen (Kintsch, 1998; Kintsch \& Kintsch, 2005). Este incremento es un proceso progresivo, que se inicia formalmente en las aulas y, por tanto, son los contextos educativos los espacios formales orientados a promover estos aprendizajes escolares. Desde esta mirada, es un factor fundamental que estos espacios de negociación y co-construcción permitan a los estudiantes apropiarse de los conocimientos y estrategias necesarias para que logren progresar en su capacidad comprensiva, actuando como andamios o ayudas dirigidas a modelar esta competencia. En este sentido y considerando la enseñanza de la comprensión de textos, una buena parte de las Actividades típicas analizadas logró establecer una diferenciación de acciones en cada una de las fases de la lectura. Al respecto, en todas ellas, independiente del tipo de calificación otorgada por los profesores, se lograron incluir tareas asociadas a cada una de las fases (Clark \& Graves, 2005; Solé, 2000); no obstante, la forma en que finalmente se secuenciaron estas tareas difirió, considerando la calificación otorgada a los profesores.

Así por ejemplo, en la fase antes de la lectura, la mayoría de los profesores desplegaron acciones dirigidas a explicitar la meta de lectura y/o activar conocimientos previos, implicando esto la creación del contexto necesario para preparar la lectura, sin embargo, una buena parte de los profesores calificados "básicos", mostró una secuenciación variable, intercalando distintas tareas sin que estas siguieran, necesariamente, una planificación lógicamente ordenada (Ortiz et al., 2007; Rincón et al., 2003; Rosas \& Jiménez, 2009).

Por otra parte, en la fase durante la lectura no se evidenciaron diferencias en el comportamiento estratégico de los profesores, dado que en esta fase se identificaron de igual modo acciones tendientes a supervisar el sentido de la lectura o solo a ejecutar la misma. Sin embargo, es importante señalar que casi la mitad de los profesores, independiente de su calificación, no propiciaron espacios para supervisar la comprensión on-line (León \& Pérez, 2000 en León, 2001) y, por tanto, facilitar que los estudiantes pudieran realizar procesos inferenciales de mayor complejidad cognitiva (Duque \& Correa, 2012; Duque \& Ovalle, 2011), logrando de esta manera supervisar su comprensión.

De igual modo, aun cuando los profesores calificados como competentes desplegaron una mayor cantidad de acciones orientadas a interpretar el sentido del texto en la fase después de la lectura, incorporando un mayor número de tareas y logrando completar la secuencia de tres acciones en la realización de las guías de trabajo, lo que supondría una mayor tendencia a retroalimentar el trabajo de los estudiantes, estrategias como la discusión o la utilización de mapas conceptuales resultaron poco frecuente en ambos grupos, implicando esto la dificultad para enseñar herramientas que permitieran facilitar el ordenamiento y jerarquización de los contenidos del texto o promover procesos de mayor complejidad (Ortiz et al., 2007; Rincón et al., 2003). En este sentido, cabe la pena preguntarse si las diferencias identificadas entre los profesores calificados como básicos y competentes, respecto a la estrategia de otorgar retroalimentación a las respuestas elaboradas por los estudiantes en las guías de trabajo, pudiera ser más bien una acción mecánica como resultado del uso de los textos escolares (Rosas \& Jiménez, 2009), más que un mecanismo que facilite efectivamente la adquisición de comportamientos estratégicos para los estudiantes (Tobias \& Everson, 2000).

A modo de cierre, aun cuando los resultados indicarían una mayor efectividad de los profesores competentes para organizar su enseñanza considerando modelos de enseñanza explícitos en comprensión de textos, se requiere profundizar más en la forma como los profesores logran negociar y co-construir el conocimiento con sus estudiantes.

\section{Referencias}

American Psychological Association. (2010). Ethical principles of psychologists and code of conduct. Recuperado de http://www.apa.org/ethics/code/index.aspx 
Anderson, R., \& Pearson, P. (1984). A schema-theoric view of basic processes in reading comprehension. En P. D. Pearson (Ed.), Handbook of reading research (pp. 255-291). New York: Longman.

Belinchón, M., Rivière, A., \& Igoa, J. M. (2000). La comprensión del discurso. En M. Belinchón, A. Rivière \& J. M. Igoa (Eds.), Psicología del lenguaje. Investigación y teoría (pp. 181-235). Madrid: Trotta.

Block, C. C., \& Pressley, M. (2002). Comprehension instruction: Research-based best practices. New York: Guilford Press.

Chall, J. (1996). Stages of reading development (2nd ed.). Fort Worth, TX: Harcourt-Brace.

Chile, Ministerio de Educación, Unidad de Currículum y Evaluación. (2004). Reglamento sobre evaluación docente. Santiago: Autor.

Chile, Ministerio de Educación, Unidad de Currículum y Evaluación. (2009a). Actualización de los objetivos fundamentales y contenidos mínimos obligatorios para el sector de lenguaje y comunicación. Santiago: Autor.

Chile, Ministerio de Educación, Unidad de Currículum y Evaluación. (2009b). Política de textos escolares. Santiago: Autor.

Clark, K., \& Graves, M. (2005). Scaffolding students' comprehension of text. The Reading Teacher, 58(6), 570-580.

Dole, J., Nokes, J. D., \& Drits, D. (2009). Cognitive strategy instruction. En G. G. Duffy \& S. E. Israel (Eds.), Handbook of research on reading comprehension (pp. 347-372). Hillsdale, NJ: Erlbaum.

Duque, C. P., \& Correa, M. (2012). Inferencias sobre un texto narrativo en contextos de interacción en la educación inicial Universitas Psychologica, 11(2), 559-570.

Duque, C. P., \& Ovalle, A. (2011). La interacción en el aula: una vía para posibilitar la comprensión inferencial de textos narrativos en niños de preescolar. Psychologia. Avances de la Disciplina, 5(2), 57-67.

Edwards, D., \& Mercer, N. (1987). Common knowledge: The development of understanding in the classroom. London: Routledge.

Rosales, J., Iturra, C., Sánchez, E. \& De Sixte, R. (2006). El análisis de la práctica educativa.Un estudio de la interacción profesor-alumnos a partir de dos sistemas de análisis diferentes. Infancia y Aprendizaje, 29(1), 65-90.

Kintsch, W. (1998). Comprehension: A paradigm for cognition. New York: Cambridge University Press.

Kintsch W., \& Kintsch, E. (2005). Comprehension. En S. G. Paris \& S. A. Stahl (Eds.), Current issues in reading comprehension and assessment (pp. 71-92). Mahwah, NJ: Erlbaum.

León, J. A. (2001). Las inferencias en la comprensión e interpretación del discurso: un análisis para su estudio e investigación. Revista Signos, 34(49-50), 113-125.

Liang, L., Peterson, C., \& Graves, M. (2005). Investigating two approaches to fostering children's comprehension of literature. Reading Psychology, 26(4-5), 387-400.

Ortiz, M., López, T., García, M., Molina, G., Pernía, E., Busto, I., ... Araujo, E. (2009). Representaciones y prácticas de docentes de educación básica en el campo de la lectura y la escritura. Letras, 51(79), 89-126.

Palincsar, A., \& Brown, A. (1984). Reciprocal teaching of comprehension fostering and comprehensionmonitoring activities. Cognition and Instruction, 1(2), 117-175.

Pressley, M., \& Afflerbach, P. (1995). Verbal protocols of reading: The nature of constructively responsive reading. Hillsdale, NJ: Erlbaum.

Rincón, G., De la Rosa, A., Chois, P., \& Niño, R. M. (2003). Entre-textos: enseñanza y aprendizaje de la comprensión de textos escritos en experiencias innovadoras de educación primaria del Valle del Cauca. Lenguaje, 31, 142-160.

Rosas, M., \& Jiménez, P. (2009). Efectividad de las estrategias de enseñanza de la comprensión de textos escritos. Un estudio de caso. Revista Signos, 42(71), 409-427.

Ruiz, J. (1999). Metodología de la Investigación Cualitativa. Bilbao: Universidad de Deusto.

Sánchez, E., García, R., \& Rosales, J. (2010). La lectura en el aula: qué se hace, qué se debe hacer y qué se puede hacer. Barcelona: Grao.

Sánchez, E., García, R., Rosales, J., De Sixte, R., \& Castellano, N. (2008). Elementos para analizar la interacción entre estudiantes y profesores: iqué ocurre cuando se consideran diferentes dimen- 
siones y diferentes unidades de análisis? Revista de Educación, 346, 105-104.

Solé, I. (2000). Estrategias de lectura. Barcelona: Graó. Stodolsky, S. (1991). La importancia del contenido en la enseñanza. Actividades en las clases de matemáticas y ciencias sociales. Barcelona: Paidós.

Tobias, S., \& Everson, H. T. (2000). Assessing metacognitive knowledge monitoring. En G. Schraw
\& J. C. Impara (Eds.), Issues in the measurement of metacognition (pp. 147-222). Lincoln, NE: Buros.

Vygotsky, L. S. (1978). Mind in society: The development of higher psychological processes. Cambridge, MA: Harvard University Press.

Wells, G. (2001). Indagación dialógica. Hacia una teoría y una práctica socioculturales de la educación. Barcelona: Paidós. 
Apéndice

Resumen de los tipos de episodios, metas y patrones generales de actuación

\begin{tabular}{|c|c|c|}
\hline Tipo episodio & Meta & $\begin{array}{l}\text { Patrón de } \\
\text { actuación }\end{array}$ \\
\hline Planificación & $\begin{array}{l}\text { Explicitar objetivos de Unidad. Explicitar objetivos de clase. } \\
\text { Explicitar objetivos de lectura. } \\
\text { Definir meta lectura. Explicitar medios para lograr el objetivo. }\end{array}$ & $\begin{array}{l}\text { Monologal } \\
\text { Diálogo }\end{array}$ \\
\hline Conocimientos previos & $\begin{array}{l}\text { Activar conocimientos de los estudiantes relacionados con: tipo de } \\
\text { textos, contenido lectura, inferencias título, anticipaciones historia, } \\
\text { elaboración hipótesis de lectura. }\end{array}$ & Diálogo \\
\hline Dictado & Dictar definiciones conceptuales. & Monologal \\
\hline Revisión tarea & $\begin{array}{l}\text { El objetivo central de este tipo de actividad típica radicaba en la } \\
\text { revisión de tareas elaboradas por los alumnos fuera de la jornada de } \\
\text { clases. }\end{array}$ & Diálogo \\
\hline Repaso concepto leyenda & $\begin{array}{l}\text { Repetir las ideas principales asociadas al concepto de leyenda como } \\
\text { parte del género narrativo. }\end{array}$ & Diálogo \\
\hline Explicación contenido & $\begin{array}{l}\text { Entregar información vinculada al nuevo contenido del texto } \\
\text { argumentativo. }\end{array}$ & Diálogo \\
\hline Organización lectura & $\begin{array}{l}\text { Entregar instrucciones de turnos de lectura o procedimientos } \\
\text { lectura. }\end{array}$ & Monologal \\
\hline Instrucciones subrayado & Clarificar estrategia de subrayado de la lectura. & Monologal \\
\hline Lectura & Ejecutar la acción de leer. & $\begin{array}{l}\text { Profesora } \\
\text { Estudiante } \\
\text { Silenciosa }\end{array}$ \\
\hline Interpretación & $\begin{array}{l}\text { Interpretar los contenidos que emergían de la lectura. Interpretar } \\
\text { las estrategias lectoras utilizadas. } \\
\text { Interpretar la estructura o partes de los textos. }\end{array}$ & $\begin{array}{l}\text { Diálogo } \\
\text { monologal }\end{array}$ \\
\hline Vocabulario & Revisar el significado de algunas palabras contenidas en los textos. & Diálogo \\
\hline Instrucciones guía de trabajo & $\begin{array}{l}\text { Entregar instrucciones y clarificar aspectos relacionados con las } \\
\text { preguntas contenidas en las guías de trabajo. }\end{array}$ & $\begin{array}{l}\text { Monologal } \\
\text { Diálogo }\end{array}$ \\
\hline Recapitulación & Síntesis de los aspectos trabajados durante la clase. & $\begin{array}{l}\text { Monologal } \\
\text { Diálogo }\end{array}$ \\
\hline $\begin{array}{l}\text { Elaboración conjunta guía de } \\
\text { trabajo }\end{array}$ & $\begin{array}{l}\text { Responder conjunta y explícitamente las preguntas de las guías de } \\
\text { trabajo. }\end{array}$ & Diálogo \\
\hline Análisis de experiencias & $\begin{array}{l}\text { Interpretación de los contenidos del texto, a partir de la revisión de } \\
\text { las experiencias particulares de los estudiantes. }\end{array}$ & Diálogo \\
\hline Desarrollo guía de trabajo aula & $\begin{array}{l}\text { Responder a las preguntas o tareas contenidas en las guías de } \\
\text { trabajo o en el libro de textos. }\end{array}$ & $\begin{array}{l}\text { C/supervi. } \\
\text { S/supervi. }\end{array}$ \\
\hline Revisión guía de trabajo aula & $\begin{array}{l}\text { Corregir las respuestas elaboradas por los estudiantes en las guías de } \\
\text { trabajo. }\end{array}$ & $\begin{array}{l}\text { Diálogo } \\
\text { Monologal }\end{array}$ \\
\hline Autoevaluación & $\begin{array}{l}\text { Responder una rúbrica vinculada con la experiencia de aprendizaje } \\
\text { y el desempeño de cada estudiante. }\end{array}$ & Diálogo \\
\hline Identificación estructura noticia & $\begin{array}{l}\text { Marcar e identificar en un periódico, las partes de un texto } \\
\text { informativo. }\end{array}$ & Diálogo \\
\hline Instrucciones tarea & $\begin{array}{l}\text { Entregar de instrucciones para el desarrollo de tareas fuera del } \\
\text { horario escolar. }\end{array}$ & Monologal \\
\hline Mapa conceptual & Elaboración de organizadores gráficos de las lecturas. & Diálogo \\
\hline $\begin{array}{l}\text { Exposición oral y revisión de guía } \\
\text { de trabajo en aula }\end{array}$ & $\begin{array}{l}\text { Exposiciones grupales acerca del resultado de algunas de las tareas } \\
\text { asignadas y retroalimentación de esas exposiciones de manera } \\
\text { pública. }\end{array}$ & Diálogo \\
\hline
\end{tabular}

Fuente: elaboración propia 
